\title{
Interactions between immune and bone cells: new insights with many remaining questions
}

\author{
Joseph Lorenzo
}

University of Connecticut Health Center, Farmington, Connecticut 06030, USA.

Phone: (860) 679-8199; Fax: (860) 679-1258; E-mail: jlorenzo@nso2.uchc.edu.

It has been over 25 years since the first observations that cells of the immune system could influence the functions of bone cells. Since that time, significant strides have been made in our understanding of the role of the immune system in bone cell activity. A number of recent discoveries demonstrated that a variety of cytokines and growth factors can influence the function of both bone and immune cells, advancing our knowledge of the interactions of these cellular systems, but important questions remain to be answered before we can fully appreciate the intricacies of the relationship between immune and bone cells.

Developmental origins of bone cells Bone cells derive from two lineages. Osteoclasts, which are responsible for bone resorption, are large multinucleated cells that are uniquely capable of removing both the organic and mineral components of bone. Osteoclasts share a common origin with cells of the monocyte/macrophage lineage and, as such, respond to and produce many of the cytokines that regulate monocyte/macrophage activities. Bone matrix is formed by osteoblasts, which originate from bone marrow stromal stem cells of mesenchymal origin. It is now well established that this marrow stromal cell can differentiate into a variety of lineages including osteoblasts, adipocytes, muscle cells, and fibroblasts (1). Marrow stromal cells produce a variety of cytokines that are critical for hematopoietic cell differentiation, and osteoblasts appear to retain some capacity to produce factors that influence the lineage development of bone marrow cells. For example, it has long been noted that there is a spatial organization to B-lymphopoiesis in the bone marrow, such that the earliest progenitors are immediately adjacent to or in intimate contact with the endosteal surface of bone, and the most mature lineages are located in the central bone marrow. This spatial organization of Blymphocyte development suggests that osteoblast lineage cells in the endosteum of bone produce factors that maintain the adjacent early B-lymphocyte precursors.

\section{Cytokines and bone homeostasis}

The first observation that immune cells could influence the activity of bone cells came from the finding that supernatants from phytohemagglutininstimulated peripheral blood monocytes of normal humans contained factors that stimulated bone resorption (2). This activity was named osteoclast activating factor $(\mathrm{OAF})$. When it was eventually purified and sequenced, the principal stimulator of bone resorption in these crude OAF preparations was found to be the cytokine IL-1 (3). In addition to its ability to stimulate osteoclast formation and resorbing activity, IL-1 is a mediator of a variety of inflammatory responses and a potent stimulus of prostaglandin synthesis, which independently increases bone resorption. It also is an inhibitor of osteoblast activity and bone formation.

Subsequent to the identification of IL-1 as a bone resorption stimulus, TNF (4) and IL-6 (5) were also found to have this activity. Like IL-1, these cytokines are critical mediators of inflammatory responses. It has now been demonstrated that a long list of cytokines can affect bone. Factors that have been identified as stimulators of resorption include IL-1, TNF, IL-6, IL11, IL-15, and IL-17. Inhibitors of resorption include IL-4, IL-10, IL-13, IL-18, and IFN- $\gamma$. TGF- $\beta$ and prostaglandins can have either stimulatory or inhibitory effects on resorption, depending on the conditions under which these factors are examined (for review see ref. 6).

Production of cytokines by immune cells has been linked to human diseases that involve bone. Perhaps the most extensive studies have been of the role of cytokines in the development of osteoporosis. Estrogen withdrawal after menopause is associated with a rapid and sustained increase in the rate at which bone is lost. This phenomenon seems to result from an increase in bone resorption that is not met by an equivalent increase in bone formation. The bone loss that occurs in susceptible individuals can be significant and result in a syndrome of pathologic fractures and accompanying morbidity. Thus, Pacifici et al. initially demonstrated that conditioned medium from cultures of peripheral blood monocytes from osteoporotic women with rapid bone turnover contained more IL-1 activity than did those from women with slow bone turnover or normal controls (7). In subsequent studies, these authors demonstrated increased production of both IL-1 and TNF by cultured peripheral blood monocytes after surgical menopause, which was not present in women who underwent surgical menopause and then received estrogen replacement. In rodents, treatment with inhibitors of IL- 1 and TNF was able to prevent the bone loss that occurred with ovariectomy. In addition, it has now been shown that ovariectomy is not associated with bone loss in mice that lack the biologically active type-1 IL-1 receptor (8) or are genetically engineered to overexpress a soluble form of TNF receptor and, hence, are incapable of responding to TNF (9). These findings strongly link the effects of estrogen withdrawal on bone loss to effects of estrogen on the production or activity of IL-1 and TNF.

The role of IL- 6 in postmenopausal bone loss has been most extensively studied in rodents. The initial observations of Jilka et al. (10) demonstrated that neutralizing antibodies to IL-6 could prevent the increase in osteoclast numbers that occurred after ovariectomy. Subsequently, it was shown that mice deficient in IL-6 failed to lose bone mass after ovariectomy (11). Produc- 
tion of IL- 6 by stromal and hematopoietic cells is inhibited by estrogen treatment, and studies using an $I L-6$ promoter-reporter construct suggest that this effect is transcriptionally mediated.

IL- 6 has also been implicated in the pathogenesis of Paget's disease of bone (12). IL-6 is produced by Pagetic patients' osteoclasts, which also express IL-6 receptors. In addition, increased levels of IL- 6 are present in the marrow plasma and serum of Pagetic patients. IL- 6 appears to also mediate the pathologic changes of Gorham-Stout disease, or massive oste- olysis (13), a rare osteolytic condition that involves extensive locally aggressive resorption of bone. Moreover, serum from these patients contains increased IL-6 levels, and the enhanced osteoclastogenesis observed when cells are treated with this serum in vitro can be inhibited by a neutralizing antibody to this cytokine. Finally, increased IL-6 production is found in the lesions of polyostotic fibrous dysplasia (14) and may mediate some of the bone pathology that is seen in this condition.

The role of cytokines in the bone disease that occurs with malignancy has also been studied extensively (15). In hematological malignancies such as lymphomas or multiple myeloma, which are associated with increased osteoclast formation and activity, a variety of cytokines, including IL-1, TNF, and IL-6, have been implicated as mediating the effects on bone. Unlike the bone disease that is associated with solid tumors and is typically mediated by parathyroid hormone-related protein (PTHrP), hematological malignancies are often characterized by an uncoupling of resorption from formation and the frequent development of
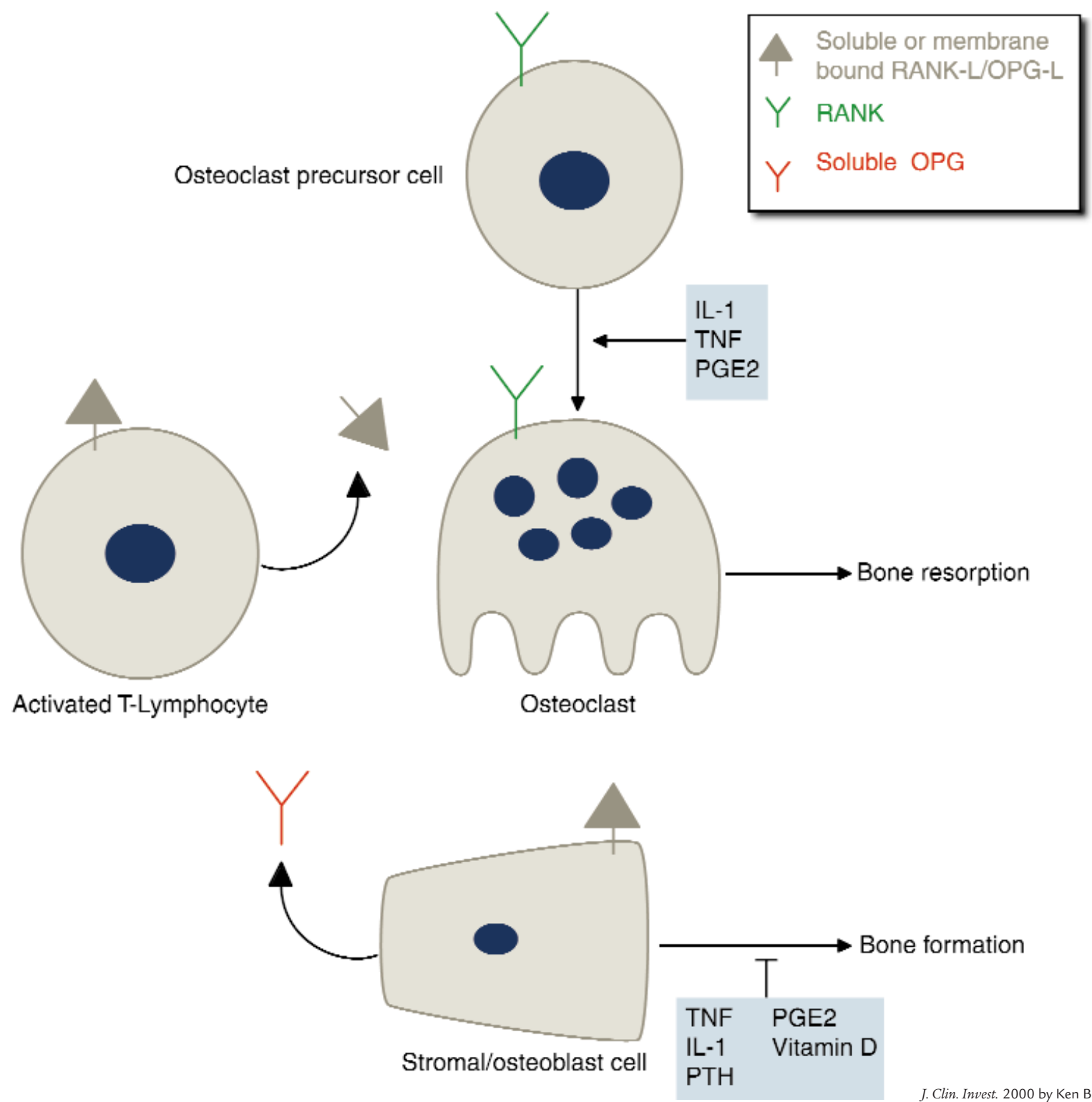

J. Clin. Invest. 2000 by Ken Beauchamp

\section{Figure 1}

Regulation of osteoclast formation. In normal physiology, osteoclast formation is regulated by production of membrane-bound RANK-L/OPG-L and soluble OPG in stromal/osteoblastic cells under the influence of stimulators of resorption. In inflammatory states, activated T lymphocytes produce both membrane-bound and soluble RANK-L/OPG-L, which stimulates RANK on osteoclast precursor cells and initiates their differentiation into osteoclasts. IL-1, TNF, and PGE2 may also directly affect osteoclast precursors or mature osteoclasts. 
purely lytic bone lesions. Significantly, both IL-1 and TNF directly affect bone by stimulating resorption and inhibiting formation.

\section{Immune functions of RANK-L and its partners}

Our understanding of the mechanism by which stimulators of resorption activate and maintain osteoclastic responses in bone was greatly advanced by the discovery of a TNFrelated ligand and two TNF receptor-related proteins. These proteins have acquired multiple names because four different groups identified them independently. The ligand, referred to here as RANK-L/OPG-L, was originally cloned and sequenced as a receptor activator of NF- $\mathrm{KB}$ ligand (RANK-L) (16) and as a TNF-related activation induced cytokine (TRANCE) (17) by investigators who were examining $\mathrm{T}$ lymphocyte products. Subsequently, two groups identified the same protein as osteoprotegerin ligand (OPGL) (18) and osteoclast differentiation factor (ODF) (19) based on its ability to stimulate osteoclast formation.

The receptor for RANK-L/OPG-L on responsive cells is named RANK (16). In addition, a variety of cells produce a soluble decoy receptor that has been termed osteoprotegerin (OPG) (20). OPG is released from cells and binds RANK-L/OPG-L, thus preventing activation of RANK. The majority of stimulators of resorption enhance the production of RANK-L/OPG-L, and some also inhibit OPG production in stromal and osteoblastic cells. Hence, regulation of RANK-L/OPG-L and OPG appears to be a critical mechanism by which stimulators of resorption influence the activity of osteoclasts in bone (see the recent JCI Commentary by F.P. Ross for a detailed review of this subject [ref. 21]).

Along with their potent effects on osteoclast development, RANKL/OPG-L, OPG, and RANK have important roles as regulators of immune cell function. This is most clearly demonstrated in RANKL/OPG-L-deficient (22) and RANKdeficient mice $(23,24)$. In addition to osteopetrosis arising from a complete absence of osteoclasts, mice of these two mutant genotypes show significant alterations in both immune cells and immune cell organs. Thymic cellularity and size are significantly decreased in RANK-L/OPG-L-deficient mice. Furthermore, differentiation of $\mathrm{CD} 44^{-} \mathrm{CD} 25^{+}$precursor cells into CD44-CD25- cells - an important step in maturation to $\mathrm{CD}^{+}$and $\mathrm{CD}^{+}$single-positive thymocytes - is blocked in the thymus of RANKL/OPG-L-deficient mice. A similar block was seen in OPG-overexpressing transgenic mice, suggesting that RANK-L/OPG-L is a critical factor in T-lymphocyte maturation in the thymus at the stage of pre-T-cell receptor expression.

An equally striking observation was that B-lymphocyte maturation in the bone marrow of RANK-L/OPG$\mathrm{L}$-deficient mice arrests at the progression of $\mathrm{B} 220^{+} \mathrm{CD} 25^{-}$pro-B lymphocyte precursors to $\mathrm{B} 220^{+} \mathrm{CD} 25^{+}$ pre-B lymphocytes. Despite the common developmental origin of osteoclasts and monocytes and the fact that RANK-L/OPG-L can act as a dendritic cell survival factor, RANK-L/OPGL-deficient mice show normal dendritic cell and monocyte development. However, $\mathrm{T}$ lymphocytes from these mice show poor induction of the cytokines IFN- $\gamma$, IL-2, IL-4, IL-5, and IL-6 upon stimulation with anti-CD3 and anti-CD28.

Perhaps the most intriguing aspect of RANK-L/OPG-L-deficient mice is their complete lack of lymph-node development, a defect that does not appear to result from a T-lymphocyte homing deficiency. Interestingly, Peyer's patches are present in RANK-L/OPG-L-deficient mice, although they are severely reduced in size. RANK-deficient mice manifest a similar phenotype, except that their thymi are of normal size and cellularity. This difference raises the interesting possibility that RANKL/OPG-L may interact with another receptor in the thymus.

In normal physiology, osteoclast formation is regulated by production of membrane-bound RANK-L/OPG-L and soluble OPG in stromal/osteoblastic cells under the influence of stimulators of resorption (Figure 1). However, RANK-L/OPG-L production by activated $\mathrm{T}$ lymphocytes can also stimulate osteoclast formation and bone resorption, and IL-1, TNF, and PGE2 can affect osteoclasts directly. Kong et al. (25) recently demonstrated that activated $T$ cells produce both membranebound and soluble RANK-L/OPG-L, which can activate osteoclastogenesis.
These authors also found that transfer of spontaneously activated $\mathrm{ctla}^{-/-} \mathrm{T}$ lymphocytes into RANK-L/OPGL-deficient mice caused bone loss, implying that $\mathrm{T}$ lymphocyte-derived RANK-L/OPG-L can directly stimulate osteoclast formation in vivo.

\section{The RANK-L/OPG-L pathway in alveolar bone loss}

In this issue of the JCI, Teng et al. (26) describe another condition in which $\mathrm{T}$ lymphocytes act through the RANKL/OPG-L pathway to promote pathological bone resorption. Localized juvenile periodontitis (LJP), a severe form of periodontitis that causes alveolar bone destruction, is caused by infection of the gingival epithelium with Actinobacillus actinomycetemcomitans (Aa). These authors transferred peripheral blood leukocytes from LJP patients into immune-deficient NOD/SCID mice and found that the human $\mathrm{CD}^{+} \mathrm{T}$ lymphocytes became activated in chimeric mice that were rechallenged with $A a$. These human cells were induced to express RANK-L/OPG-L, resulting in alveolar bone loss in the murine host.

These findings, together with those of Kong et al. (25), suggest that T lymphocyte-derived RANK-L/OPG-L is a mediator of the osteoclast formation and bone loss that is seen in LJP. Teng et al. (26) show that treatment of the chimeric mice with OPG ameliorates the bone loss after $A$ a rechallenge, providing strong evidence that RANKL/OPG-L contributes to bone resorption in LJP. However, it is unclear whether $\mathrm{T}$ lymphocytes are the only source of this factor, since stromal or osteoblastic cells in the alveolar bone can also produce RANK-L/OPG-L in response to inflammatory cytokines generated after Aa rechallenge. Most likely, both T-lymphocyte and stro$\mathrm{mal} /$ osteoblastic production of RANK$\mathrm{L} / \mathrm{OPG}-\mathrm{L}$ are involved in the alveolar bone loss of LJP, but direct evidence regarding the source of this factor will require in situ hybridization and immunohistochemical studies of bone from affected individuals.

The finding that $\mathrm{T}$ lymphocytes are capable of producing RANK-L/OPG-L, which can stimulate osteoclastogenesis, implies that this mechanism represents an additional pathway by which the bone and immune systems interact. Significantly, Kong et al. (25) reported that six of seven T-cell lymphomas from mice 
express high levels of RANK-L/OPG-L on their cell surfaces. This finding implies that $\mathrm{T}$ lymphocyte-derived RANK-L/OPG-L may also cause the bone pathology that is sometimes seen with human T-cell lymphomas. This interesting hypothesis awaits additional studies for confirmation.

1. Prockop, D.J. 1997. Marrow stromal cells as stem cells for nonhematopoietic tissues. Science. 276:71-74

2. Horton, J.E., Raisz, L.G., Simmons, H.A., Oppenheim, J.J., and Mergenhagen, S.E. 1972. Bone resorbing activity in supernatant fluid from cultured human peripheral blood leukocytes. Science 177:793-795.

3. Dewhirst, F.E., Stashenko, P.P., Mole, J.E., and Tsurumachi, T. 1985. Purification and partial sequence of human osteoclast-activating factor: identity with interleukin 1 beta. J. Immunol. 135:2562-2568.

4. Bertolini, D.R., Nedwin, G.E., Bringman, T.S. Smith, D.D., and Mundy, G.R. 1986. Stimulation of bone resorption and inhibition of bone formation in vitro by human tumour necrosis factors. Nature. 319:516-518

5. Ishimi, Y., et al. 1990. IL-6 is produced by osteoblasts and induces bone resorption. J. Immunol. 145:3297-3303.

6. Horowitz, M.C., and Lorenzo, J. 1996. Local regulators of bone: IL-1, TNF, and lymphotoxin, interferon gamma, IL-8, IL-10, IL-4, the LIF/IL6 family and additional cytokines. In Principals of bone biology. J. Bilezikian, L. Raisz, and G.
Rodan, editors. Academic Press. San Diego, California, USA. 687-700.

7. Pacifici, R., et al. 1987. Spontaneous release of interleukin 1 from human blood monocytes reflects bone formation in idiopathic osteoporosis. Proc. Natl. Acad. Sci. USA. 84:4616-4620.

8. Lorenzo, J.A., et al. 1998. Mice lacking the type I interleukin-1 receptor do not lose bone mass after ovariectomy. Endocrinology. 139:3022-3025.

9.Ammann, P., et al. 1997. Transgenic mice expressing soluble tumor necrosis factor-receptor are protected against bone loss caused by estrogen deficiency. J. Clin. Invest. 99:1699-1703.

10. Jilka, R.L., et al. 1992. Increased osteoclast development after estrogen loss: mediation by interleukin-6. Science. 257:88-91.

11. Poli, V., et al. 1994. Interleukin-6 deficient mice are protected from bone loss caused by estrogen depletion. EMBO J. 13:1189-1196.

12. Roodman, G.D., et al. 1992. Interleukin 6. A potential autocrine/paracrine factor in Paget's disease of bone. J. Clin. Invest. 89:46-52

13. Devlin, R.D., Bone, H.G., III, and Roodman, G.D. 1996. Interleukin-6: a potential mediator of the massive osteolysis in patients with Gorham-Stout disease. J. Clin. Endocrinol. Metab. 81:1893-1897.

14. Yamamoto, T., et al. 1996. Increased IL-6 production by cells isolated from the fibrous bone dysplasia tissues in patients with McCuneAlbright syndrome. J. Clin. Invest. 98:30-35.

15. Guise, T.A., and Mundy, G.R. 1998. Cancer and bone. Endocr. Rev. 19:18-54.

16. Anderson, D.M., et al. 1997. A homologue of the TNF receptor and its ligand enhance T-cell growth and dendritic-cell function. Nature. 390:175-179.

17. Wong, B.R., et al. 1997. TRANCE is a novel lig- and of the tumor necrosis factor receptor family that activates c-Jun $\mathrm{N}$-terminal kinase in $\mathrm{T}$ cells. J. Biol. Chem. 272:25190-25194.

18. Lacey, D.L., et al. 1998. Osteoprotegerin ligand is a cytokine that regulates osteoclast differentiation and activation. Cell. 93:165-176.

19. Yasuda, H., et al. 1998. Osteoclast differentiation factor is a ligand for osteoprotegerin osteoclastogenesis-inhibitory factor and is identical to TRANCE/RANKL. Proc. Natl. Acad. Sci. USA. 95:3597-3602.

20. Simonet, W.S., et al. 1997. Osteoprotegerin: a novel secreted protein involved in the regulation of bone density. Cell. 89:309-319.

21. Ross, F.P. 2000. RANKing the importance of measles virus in Paget's disease. J. Clin. Invest. 105:555-558.

22. Kong, Y.Y., et al. 1999. OPGL is a key regulator of osteoclastogenesis, lymphocyte development and lymph-node organogenesis. Nature. 397:315-323.

23. Dougall, W.C., et al. 1999. RANK is essential for osteoclast and lymph node development. Genes Dev. 13:2412-2424.

24. Li, J., et al. 2000. RANK is the intrinsic hematopoietic cell surface receptor that controls osteoclastogenesis and regulation of bone mass and calcium metabolism. Proc. Natl. Acad. Sci. USA. 97:1566-1571.

25. Kong, Y.Y., et al. 1999. Activated T cells regulate bone loss and joint destruction in adjuvant arthritis through osteoprotegerin ligand. Nature. 402:304-309.

26. Teng, Y.-T.A., et al. 2000. Functional human Tcell immunity and osteoprotegerin ligand (OPG-L) control alveolar bone destruction in periodontal infection. J. Clin. Invest. 106:R59-R67. 